Revue d'histoire de l'Amérique française

- REVUE D.HISTOIRE DE L'AMÉRIQUE FRANÇAISE

\title{
Inventaire général des registres des archives de la Chancellerie de l’Archevêché de Montréal 1643-1876
}

\section{François Beaudin}

Volume 20, numéro 4, mars 1967

URI : https://id.erudit.org/iderudit/302638ar

DOI : https://doi.org/10.7202/302638ar

Aller au sommaire du numéro

Éditeur(s)

Institut d'histoire de l'Amérique française

ISSN

0035-2357 (imprimé)

1492-1383 (numérique)

Découvrir la revue

Citer ce document

Beaudin, F. (1967). Inventaire général des registres des archives de la Chancellerie de l'Archevêché de Montréal 1643-1876. Revue d'histoire de l'Amérique française, 20(4), 669-700. https://doi.org/10.7202/302638ar d'utilisation que vous pouvez consulter en ligne. 


\section{INVENTAIRE D'ARCHIVES}

\section{INVENTAIRE GÉNÉRAL DES REGISTRES DES ARCHIVES DE LA CHANCELLERIE DE L'ARCHEVÊCHÉ DE MONTRÉAL *}

\section{$1643 \cdot 1876$}

Dans une précédente livraison de cette revue ${ }^{1}$, nous signalions, que le dépôt des Archives de la Chancellerie de l'Archevêché de Montréal comporte quatre secteurs de documents, à savoir: des registres, des dossiers contenant des pièces détachées, des photos et des cartes géographiques ou plans.

Comme on le sait, le secteur des dossiers fait l'objet d'inventaires en cours de parution dans cette revue ${ }^{2}$. Le contenu de ces dossiers est donc ainsi mis à la disposition des chercheurs et le travail se poursuit selon un rythme régulier.

Quant au secteur des registres, il a déjà joui d'une présentation d'ensemble dans un article du Père Gaston Carrière, o.m.i. ${ }^{3}$. Ici même, en mars 1966, nous disions à ce sujet: "On trouvera dans la conférence du Père Carrière mentionnée ci-haut quelques indications. Elles ne peuvent malheureusement donner qu'une faible idée de la richesse de notre dépôt en ce domaine. On y reviendra, espérons-le, dans un article subséquent." " Seul, en effet, un inventaire général des registres conservés en notre dépôt d'archives peut fournir aux chercheurs

\footnotetext{
*Voir notre Revue, XIX, no 4 (mars 1966) : 652-664; XX, no 1 (juin 1966): 146-166; no 2 (sept. 1966): 322-341.

1 RHAF, XIX, no 4 (mars 1966): 654

2 Dans la livraison de mars 1966, aux pp. 652-664 incl., on a présenté l'inventaire général des dossiers pour les années 1600-1760 et 1760-1835. Dans la livraison de juin 1966, aux pp. 146-166 incl., on a présenté l'inventaire général des dossiers pour les années 1836-1876. Dans la livraison de septembre 1966, aux pp. 322-341 incl., on a présenté l'inventaire analytique des dossiers pour la période 1600-1760. La parution de l'inventaire analytique de la période 17601835 débutera probablement dans une prochaine livraison de cette revue.

3 "Les Richesses inexploitées de nos Archives diocésaines", in Revue de l'Université d'Ottawa, vol. 34 (1964): 73-96 et La Société canadienne d'Histoire de lEglise catholique - Rapport 1963: 63-81. Dans ce rapport, le paragraphe concernant notre dépôt se trouve aux pp. 69 et 70.
}

4 RHAF, XIX, no 4 (mars 1966): 654. 
les indications complètes quant au contenu de notre dépôt en ce domaine. C'est ce que nous allons présenter maintenant.

Signalons immédiatement que nous mentionnerons tous les registres qui contiennent des documents dont le sujet est relatif à la période antérieure à 1876 , que ce soit des originaux ou des copies. Le plus vieux de ces documents enregistrés est la transcription de "Les Véritables Motifs de Messieurs et Dames de la Société de Notre-Dame de Montréal pour la conversion des Sauvages de la Nouvelle-France", faite par l'abbé J. Desautels, curé, au siècle dernier.

Dans le cas où le registre comporte des documents datant d'après 1876, nous l'indiquons et nous donnons le nombre des pages couvrant la période antérieure à 1876 . Les documents datant d'après 1876 ne sont pas accessibles aux chercheurs: en effet, la date limite de consultation est 1876 en notre dépôt d'archives. Pour ces raisons, les dates extrêmes de cet inventaire sont donc 1643 et 1876.

Avec la parution du présent inventaire, les chercheurs ont maintenant en main l'inventaire général et des dossiers et des registres des Archives de la Chancellerie de l'Archevêché de Montréal.

Nous entendons désormais poursuivre notre travail de publication dans le domaine des inventaires analytiques. Pour ce qui est des dossiers, ils paraitront, lorsque prêts, dans cette Revue, si l'on continue de nous y donner l'hospitalité, ce que j'espère, et pour ce qui est des registres, leurs inventaires analytiques continueront de paraitre dans le Rapport de l'Archiviste du Québec.

C'est avec joie que nous livrons aux historiens le fruit de quatre années de labeur.

Archevêché de Montréal,

Françors Beaudin, ptre 10 mars 1967. 


\section{REGISTRES CONSERVÉS AUX ARCHIVES \\ DE LA CHANCELLERIE DE L'ARCHEVECHE DE MONTRÉAL}

\section{TABLE DES SÉRIES}

RL - Registres de lettres

Sous-série RLL - Registres de lettres de Mgr Lartigue

RLB - Registres de lettres de Mgr Bourget

RLF - Registres de lettres de Mgr Fabre

RC - Registres de la Chancellerie

RD - Registres de Décrets

RNLP - Registres de nominations - Lettres de pouvoir

RCO - Registres de Confirmations

RDM - Registres de Dispenses de Mariages

RCC - Registres du Chapitre de la Cathédrale

RG - Registres du Gésu

REC - Registres de l'état civil Sous-série REC 1 - Registres de la paroisse de Repentigny

RCD - Registres et cahiers divers

\section{SÉRIES RL ET RC}

Introduction. - Dans ces deux séries, les seules sous-séries RLL, RLB et la série RC font l'objet d'un inventaire analytique qui paraît dans le Rapport de l'Archiviste du Québec depuis 1941. L'inventaire de la série RLF n'a pas encore paru. Dans ces Rapports, on présente un inventaire analytique de la série RLL ou RLB et on intercale dans cet inventaire l'inventaire analytique de la série RC, par ordre chronologique.

Pour évaluer l'importance de ces registres, il suffit de montrer comment l'administration diocésaine de l'époque avait résolu le problème de la classification des documents.

Les documents reçus des individus ou des institutions étaient collés par la marge dans des cahiers à onglets. Chaque institution possédait son cahier, dans les archives diocésaines. Aux Archives de la Chancellerie de l'Archevêché de Montréal, tous ces cahiers, sauf quelquesuns conservés à titre de specimen, ont été défaits, en 1950, et les 
documents ont été déposés dans des chemises. On travaille actuellement à décoller les onglets des documents originaux. Ce sont ces pièces détachées qui font l'objet des inventaires analytiques en cours de parution dans cette Revue.

Les réponses à ces lettres, quant à elles, étaient consignées par le secrétaire de l'évêque dans un registre de lettres, s'il s'agissait d'une simple correspondance (sous-séries RLL - RLB - RLF), ou dans un registre de la chancellerie, s'il s'agissait d'un acte officiel (série RC). On y inscrivait aussi, dans l'un comme dans l'autre registre, le texte des lettres ou des actes officiels que l'évêque prenait l'initiative d'envoyer.

Or, on connaît la loi ecclésiastique qui demande de verser aux diocèses nouvellement crées les archives des institutions faisant partie du nouveau territoire constitué en diocèse.

Il arrive donc ceci comme conséquence. Nos archives ne possèdent plus les cahiers à onglets concernant des territoires faisant maintenant partie d'autres diocèses que Montréal, mais qui lui étaient originellement rattachés: ces cahiers, en effet, ont été envoyés aux archives des nouveaux évêchés, lorsque des diocèses nouveaux furent créés. Mais nos archives gardent encore, cependant, dans ces deux séries de registres (RL et $\mathbf{R C}$ ), toutes les réponses et décisions des évêques du temps, non seulement pour le territoire actuel du diocèse de Montréal, mais encore pour les territoires qui en ont été détachés, durant la période où ils en faisaient partie.

On voit ainsi toute la richesse de contenu de ces registres et on comprend pourquoi ils ont été les premiers à faire l'objet d'inventaires analytiques.

Nous donnerons tout d'abord l'inventaire général des sous-séries RLL - RLB - RLF et de la série RC, puis la liste des inventaires analytiques parus à ce jour dans le Rapport de l'Archiviste du Québec.

\section{SÉRIE RL - REGISTRES DE LETTRES \\ SOUS-SÉRIE RLL - REGISTRES DE LETTRES DE Mgr J.-J. LARTIGUE}

RLL 1 - 20 septembre 1819 au 23 mars 1822 :

1. Table des lettres de Mgr Lartigue, adressées à Mgr Plessis avant qu'il ait été consacré évêque de Telmesse et dont les originaux se trouvent aux archives du secrétariat de l'Archevêché de Québec. (1819 - 20 sept.. 30 oct.; $1820-2$ mars, 11 avril, ler sept., 14 sept., 30 oct., 25 nov., 25 déc., 31 déc.). -1 p.

2. Analyse des lettres ci-dessus mentionnées. Cette analyse fut faite par Mgr Ignace Bourget, alors qu'il était secrétaire de Mgr J.-J. Lartigue. p. 1-22. 
3. Texte des lettres ci-dessus mentionnées. Copies collationnées aux Archives de l'Archevêché de Québec par C. A. Collet, ptre. - p. (1)-(34).

4. Table alphabétique des lettres contenues dans ce volume. $-2 \mathrm{p}$.

5. Titre: "Registre des lettres écrites par Monseigneur l'Evêque de Telmesse, depuis le trente du mois de janvier, mil huit cent vingt-un jusqu'au vingttrois de mars mil huit cent vingt-deux. L. M. Quintal, S.D. eccl.55 - 1 p.

6. Texte des lettres datées entre le 30 janvier 1821 et le 23 mars 1822 . p. 1-208.

7. Table analytique des matières contenues dans ce volume. - p. 1-34.

RLL 2 - 25 mars 1822 au 24 février 1824 :

1. Table alphabétique des lettres contenues dans ce volume. - p. 1-6.

2. Titre: "Registre des lettres écrites par Mon Seigneur l'Evêque de Telmesse, depuis le vingt-cinq de Mars, Mil huit cent vingt-deux, jusqu'au vingtquatre de Février, Mil huit cent vingt-quatre. Ig. Bourget, Dcre. Secr.". En sous-titre: "Tom. 2". $-1 \mathrm{p}$.

3. Texte des lettres. - p. 1-349.

\section{RLL 3 - 24 février 1824 au ler octobre 1825 :}

1. Table alphabétique des lettres contenues dans ce volume. - p. 1-4.

2. Titre : "Registre des Lettres écrites par Mon Seigneur l'Evêque de Telmesse, depuis le vingt-quatre de Février, Mil huit cent vingt-quatre, jusqu'au premier d'Octobre, Mil huit cent vingt-cinq. Ig. Bourget Ptre. Secr.". En sous-titre: "Tom. 3". -1 p.

3. Texte des lettres. - p. 1-267.

\section{RLL 4 - ler octobre 1825 au 4 décembre 1828 :}

1. Table alphabétique des Lettres contenues dans ce volume. $-5 \mathrm{p}$.

2. Titre: "Régistre des Lettres écrites par Mon Seigneur l'Evêque de Telmesse, depuis le premier d'Octobre, mil huit cent vingt-cinq, jusqu'zu quatre Décembre, Mil huit cent vingt-huit. Ig. Bourget Ptre. Secr.". En sous-titre: "Tom. 4". - 5e p. de la table.

3. Texte des lettres. - p. 1-446.

\section{RLL 5 - 10 décembre 1828 au 5 mai 1831 :}

1. Table alphabétique des Lettres contenues dans ce Volume. - 5 p.

2. Titre: "Régistre des Lettres écrites par Mon Seigneur l'Evêque de Telmesse, depuis le dix de Décembre Mil huit cent vingt-huit, jusqu'au cinq de Mai Mil huit cent trente-un, inclusivement. Ig. Bourget Ptre. Secr.". En sous-titre: "Tom. 5". $-1 \mathrm{p}$.

3. Texte des lettres. - p. 1-454.

\section{RLL 6 - 7 mai 1831 au 28 novembre 1832 :}

1. Table alphabétique des Lettres contenues dans ce Volume. - 12 p.

2. Titre: "Registre des Lettres écrites par Mon Seigneur l'Evêque de Telmesse, depuis le sept de Mai Mil huit cent trente-un, jusqu'au vingt-huit de Novembre Mil huit cent trente-deux, inclusivement. Ig. Bourget Ptre. Secr.". En sous-titre: "Tom. 6". - 1 p.

3. Texte des lettres. - p. 1-501. 
RLL 7 - ler décembre 1832 au 27 octobre 1835 :

1. Table des Lettres contenues dans le 70 Tome. -12 p.

2. Titre: "Régistre des Lettres écrites par Mon Seigneur l'Evêque de Telmesse, depuis le premier Décembre Mil huit cent trente-deux, jusqu'au vingt-sept Octobre, Mil huit cent trente-cinq. Ig. Bourget Ptre. Secr.". En sous-titre: "Tom. 7". - p. non numérotée après la table.

3. Texte des lettres. - p. 1-844.

RLL 8 - 30 octobre 1835 au 8 décembre 1837 :

1. Table des Lettres contenues dans le 8 Tome. $-8 \mathrm{p}$.

2. Titre: "Régistre des Lettres écrites par Mon Seigneur l'Evêque de Telmesse, depuis le trente Octobre, Mil huit cent trente-cinq, jusqu'au vingtneuf de Novembre Mil huit cent trente-sept, inclusivement. Ig. Bourget Ptre. Secr.". En sous-titre: "Tom. 8". - p. 1.

3. Texte des lettres. - p. 3-457.

RLL 9 - 10 décembre 1837 au 7 avril 1840

suivi de: Relation du Voyage de l'Évêque de Montréal en Europe :

1. Table des matières contenues dans ce Volume $(A-N) .-10 \mathrm{p}$.

2. Titre: "Régistre des Lettres écrites par Mon Seigneur l'Evêque de Montréal, depuis le dix de Décembre Mil huit cent trente-sept, jusqu'au 7 Avril 1840, avec diverses pièces relatives au voyage du second Evêque à Rome. A. F. Truteau Ptre. Secrétaire.". En sous-titre: "Tom. 9". - p. 1.

3. Texte des lettres. - p. 3-285.

4. "Relation du Voyage de l'Evêque de Montréal en Europe (1841)". - p. 287-559. La transcription dactylographiée de ce texte se trouve dans RCD 109 (voir cette série, plus loin).

5. Suite de la Table des Matières (O-Y). $-4 \mathrm{p}$.

\section{SOUS-SERRIE RLB - REGISTRES DES LETTRES DE MGR BOURGET}

N.B. - Les Tomes 12 à 25 de cette sous-série étaient en mauvais état de conservation. Ils ont été transcrits à la main en 1964 et ces transcriptions sont actuellement en train d'être mises au dactylo.

RIB 1 - 19 mai 1837 - 14 octobre 1839 :

1. Titre: "Registre de lettres écrites par le coadjuteur de Montréal depuis le 19 mai 1837, jusqu'au 14 octobre, 1839.

Tome premier."

Ig. Bourget, Coad.

2. Texte des lettres. - p. 1-357, les 2 dernières feuilles non numérotées.

3. Table alphabétique. -10 p. non numérotées.

RLB 2 - 20 octobre 1839 - 4 mars 1843 :

1. Titre: "Régistre de lettres écrites par le Coadjuteur de Montréal, depuis le 20 octobre, 1839, jusqu'au 4 mars 1843.

Tome second."

† Ig. Ev. de Telmesse.

3. Table alphabétique. -30 p. non numérotées. 
RLB 3 - 9 mars 1843 - 29 juin 1845 :

1. Titre: "Régistre des lettres écrites par Monseigneur Ignace Bourget, Ev. de Montréal, depuis le 9 mars 1843, jusqu'au 29 juin 1845. Tome III."

2. Textes des lettres. - p. 1-633.

3. Table des matières. -16 p. non numérotées.

RLB 4 - 5 juillet 1845 - 12 septembre 1848 :

1. Titre: "Régistre de Lettres écrites par Monseigneur Ignace Bourget, Evêque de Montréal, depuis le 5 Juillet mil huit cent quarante cinq, jusqu'au douze septembre mil-huit-cent-quarante huit."

2. Texte des lettres. - p. 1-565.

3. Table alphabétique des Lettres contenues dans ce Volume. - p. 566-574, et $9 \mathrm{p}$. non numérotées.

\section{RLB 5 - 13 septembre 1848 - 16 février 1850 :}

1. Titre: "Régistre des Lettres écrites par Monseigneur Ignace Bourget, depuis le treize septembre mil-huit-cent quarante-huit jusqu'au seize février Mil huit cent cinquante."

2. Texte des lettres. - p. 1-446.

3. "Table des lettres contenues dans ce volume XIV". - 15 pages non numérotées. - Ce volume est appelé XIV parce qu'une ancienne numérotation additionnait les cinq registres jusqu'ici présentés de cette sous-série RLB aux neuf de la sous-série RLL précédemment présentés.

\section{RLB 6 - 28 février 1850 - 14 octobre 1851 :}

1. Titre: "Régistre des Lettres écrites par Monseigneur Ignace Bourget, Evêque de Montréal, depuis le vingt-huit Février mil huit cent cinquante jusqu'au quinze octobre mil huit cent cinquante-un".

2. Texte des lettres. - p. 1-565 et dernière page non numérotée. (On trouve aux deux dernières pages un post-scriptum du 23 octobre.)

3. "Table des lettres contenues dans ce volume". 18 p. non numérotées.

RLB 7 - 15 octobre 1851 - 24 mars 1853 :

1. Titre: "Régistre des Lettres écrites par Monseigneur Ignace Bourget, Evêque de Montréal, depuis le quinze Octobre mil huit cent cinquante-un jusqu'au vingt-quatre Mars mil huit cent cinquante-trois."

2. Texte des lettres. - p. 1-642. (Entre la p. 402 et 403, un petit cahier de 20 pages porte sur 11 pages les huit lettres écrites du 14 au 28 juillet 1852.)

3. "Table des Lettres contenues dans ce Volume". - p. 643-652, suivies de 12 pages non numérotées, suivies de la p. 653 et de 2 pages non numérotées.

RLB 8 - 16 mars 1853 - 22 octobre 1854 :

1. Titre: "Régistre des lettres écrites par Monseigneur Ignace Bourget, Evêque de Montréal, depuis le Seize Mars mil huit cent cinquante-trois jusqu'au 22 octobre mil huit cent cinquante quatre."

2. Texte des lettres. - p. 1-532.

3. "Table Alphabétique des Lettres contenues dans le Tome XVII." - p. 533-553. - Ce volume est appelé XVII parce qu'une ancienne numérotation additionnait les huit registres jusqu'ici présentés de cette sous-série RLB aux neuf de la sous-série RLL précédemment présentés. 
RLB 9 - 24 octobre 1854 - 17 décembre 1856 :

1. Titre: "Régistre des Lettres écrites par Monseigneur Ignace Bourget, Evêque de Montréal, depuis le vingt-quatre Octobre mil huit cent cinquantequatre jusqu'au dix-sept Décembre mil huit cent cinquante-six."

2. Texte des lettres. - p. 1-484.

3. Table alphabétique. $-12 \mathrm{p}$. non numérotées.

RLB 10 - 13 décembre 1856 - 18 septembre 1859 :

1. Sans titre.

2. Texte des lettres. - p. 1-599.

3. "Table des matières contenues dans le Tome XIX." - nouvelle numérotation, p. 1-27. - Ce volume est appelé XIX parce qu'une ancienne numérotation additionnait les dix registres jusqu'ici présentés de cette sous-série RLB aux neuf de la sous-série RLL précédemment présentés.

RLB 11 - 18 septembre 1859 - 17 juin 1861 :

1. Titre: "18 septembre 1859 au 17 juin $1861 . "$

2. Texte des lettres. - p. 1-458.

3. "Table des matières contenues dans ce volume. - Tome XX - Lettres." 16 pages non numérotées. - Ce volume est appelé XX parce qu'une ancienne numérotation additionnait les onze registres jusqu'ici présentés de cette sous-série RLB aux neuf de la sous-série RLL précédemment présentés.

RLB 12 - 7 juin 1861 - 29 mai 1863 :

1. Titre: "Du 7 juin 1861 au 29 mai 1863."

2. Index alphabétique. -22 pages non numérotées.

3. Texte des lettres. - p. 1-1009.

RLB 13 - 8 juillet 1863 - 6 septembre 1864 :

1. Titre: "Du 8 juillet 1863 au 6 septembre 1864."

2. Index alphabétique. -14 p. non numérotées.

3. Texte des lettres. - p. 1-698.

RLB 14 - 6 septembre 1864 - 8 mars 1866 :

1. Titre: "6 septembre 1864 au 8 mars $1866 . "$

2. Index alphabétique. -11 p. non numérotées.

3. Texte des lettres. - p. 1-525.

RLB 15 - 9 mars 1866 - 8 février 1867 :

1. Sans titre.

2. Index alphabétique. - 13 p. non numérotées.

3. Texte des lettres. - p. 1-489.

RLB 16 - 8 février 1867 - 4 novembre 1867 :

1. Titre: "Du 8 Février 1867 au 4 novembre 1867."

2. Index alphabétique. - 11 p. non numérotées.

3. Texte des lettres. - p. 1-496. 
RLB 17 - 5 novembre $1867 \cdot 12$ août 1868 :

1. Sans titre.

2. Index alphabétique. - 16 p. non numérotées.

3. Texte des lettres. - p. 1-499.

RLB 18 - 12 août 1868 - 20 mai 1870 :

1. Titre: "Du 12 août 1868 au 20 Mai 1870."

2. Index alphabétique. -19 p. non numérotées.

3. Texte des lettres. - p. 1-495.

RLB 19 - 21 mai $1870-27$ avril 1872 :

1. Sans titre.

2. Index alphabétique. -23 p. non numérotées.

3. Texte des lettres. - p. 2-959.

RLB 20 - 29 avril 1872 - 27 décembre 1872 :

1. Titre: "29 avril 1872 au 27 Décembre 1872."

2. Index alphabétique. - 16 p. non numérotées.

3. Texte des lettres. - p. 1-494.

RLB 21 - 28 décembre 1872 - 12 novembre 1873 :

1. Titre: "Du 28 Décembre 1872 au 12 novembre 1873."

2. Index alphabétique. -22 pages non numérotées.

3. Texte des lettres. - p. 1-780.

RLB 22 - 11 novembre 1873 - 17 octobre 1874:

1. Titre: "Du 11 Novembre 1873 au 17 octobre 1874."

2. Index alphabétique. $-20 \mathrm{p}$. non numérotées.

3. Texte des lettres. - p. 1-719.

RLB 23 - 16 octobre 1874 - 30 septembre 1875 :

1. Titre: "Du 16 Octobre 1874 au 30 Septembre 1875."

2. Index alphabétique. $-20 \mathrm{p}$. non numérotées.

3. Texte des lettres. - p. 1-711.

RLB 24 - 1er octobre 1875 - 23 juin 1875 :

1. Titre: "Du 1er Octobre 1875 au 23 juin 1876."

2. Index alphabétique. - $18 \mathrm{p}$. non numérotées.

3. Texte des lettres. - p. 1-715.

RLB 25 - 23 juin 1876 - 30 octobre 1880 :

1. Titre: "Du 23 Juin 1876 au 30 Octobre 1880."

2. Index alphabétique. $-18 \mathrm{p}$. non numérotées.

3. Texte des lettres de Mgr Bourget, 23 juin au 8 septembre 1876: p. 1-82.

4. Texte des lettres de Mgr Fabre, 19 septembre 1876 au 30 octobre 1880: p. 83-709. (Cette partie du registre n'est pas accessible aux chercheurs.) 


\section{SOUS-SÉRIE RLF - REGISTRES DE LETTRES DE Mgr FABRE}

\section{RLF 3 - Notre-Dame - Université - Décrets - Lettres : 22 décembre 1865; 16 juillet 1872 au 12 août 1880 :}

1. Décrets et Lettres. - f. 2 à 138 v. - [Avant 1877: passim].

2. Table des matières des copies de lettres ou autres documents - f. 139$139 \mathrm{v}$.

3. "Table des matières des Lettres de MonSeigneur E. C. Fabre, Evêque de Montréal". - f. 139 v - 140 v.

4. Broché au feuillet 143e: un cahier de 43 pages contenant les lettres enregistrées pendant l'administration de M. H. Moreau, chanoine: du 6 août 1879 au 3 février 1880 .

5. Table des matières de ce cahier. -2 p.

RLF 4 - Actes de Délibérations, en Conseil, pour affaires diocésaines Lettres :

1. “Actes de Délibérations en Conseil, pour affaires Diocésaines". - du 2 septembre 1856 au 5 novembre $1856 ; 12$ et 14 mars 1867 . - p. 4-12.

2. Texte des lettres: du 22 juillet 1880 au 30 décembre 1882. - p. 1-324. (2e pagination.)

3. Liste des prêtres qui ont souscrit des requêtes contre le Bill de Laval cette liste a été faite par M. A. Labelle, ptre, curé de St-Jérôme. - 4 juin $1881-1 \mathrm{p}$.

4. Index alphabétique des lettres. $-23 \mathrm{p}$.

RLF 9 - Lettres aux communautés du 3 avril 1874 au 15 février 1896:

1. Index: p. 1-21.

2. Texte des lettres: f. 2-104. [Avant 1877: f. 2-12 v.].

\section{SÉRIE RC - REGISTRES DE LA CHANCELLERIE}

RC 1 - 30 avril 1791 au 28 mars 1829 :

1. Titre: "Régistre Authentique des pièces et actes qui concernent le District Episcopal de Montréal, sous la juridiction de Monseigneur L'Evêque de Telmesse, Suffragant et Vicaire Général du Diocèse de Québec: le dit Registre contenant deux cents quatorze feuillets cottés et paraphés, depuis le premier jusqu'au dernier par le dit Seigneur, Evêque de Telmesse, à Montréal, le vingt un janvier, mil huit cent-vingt-un.

$\dagger$ Jean-Jacques, Ev. de Telmesse,

Par Monseigneur

2. Texte des Actes - f. 2-200.

Jos. Gaboury, Dcre. Secret. ad hoc". - f. 1.

3. "Table des actes et pièces contenus dans le premier volume des Archives du District Episcopal de Montréal". - f. 200 v. - 209.

4. Extrait de l'Ordonnance du 30 avril, 1791, pour la formation des paroisses et pour la construction et la réparation des Eglises, Presbytères et Cimetières. - dernier $f$. $v$. 
RC 2 - 1er mai 1829 - 1er mars 1837 :

1. Liste d'indults. $-1 \mathrm{p}$.

2. Titre: "Registre authentique des pièces et actes qui concernent le District Episcopal, de Mont-réal, sous la juridiction de Monseigneur l'Evêque de Telmesse, Suffragant, Auxiliaire, et Vicaire-Général du Diocèse de Québec: le dit Registre contenant deux cents-onze feuillets, cottés et paraphés, depuis le premier jusqu'au dernier, par le dit Seigneur, Evêque de Telmesse, à Mont-réal, le premier de mai, Mil huit cent vingt-neuf. T. 2

3. Texte des Actes. f. 2-203.

Ig. Bourget, ptre. secr.". - f. 1.

4. "Table des Actes et Pièces contenus dans le second volume des Archives du District Episcopal et Diocèse de Montréal". - f. 203 v. - 210 v.

RC 3 - 11 mars 1837 au 25 novembre 1840 :

1. Titre: "Registre authentique des pièces et actes qui concernent le Diocèse de Montréal, contenant deux cent deux feuillets, cottés et paraphés, depuis le premier jusqu'au dernier, par nous soussigné l'un des Vicaires Généraux de Monseigneur J. J. Lartigue, Evêque du dit Montréal, le vingt-huit février mil huit cent trente-sept.

Tome 3". - f. 1 .

Ig. Bourget, Vic. Gén. -

2. Texte des Actes. - f. 2-188 v.

3. "Table des Actes et pièces contenus dans le troisième volume des Archives du District Episcopal et Diocèse de Montréal". - f. 188-194 v.

4. Autels privilégiés en vertu d'un Indult du 31 mai 1840 qui permet à l'Evêque de Montréal d'en privilégier trente. - dernier folio.

RC 4 - 30 novembre 1840 au 30 mai 1843 :

1. Titre: "Registre authentique des pièces et actes qui concernent le Diocèse de Montréal contenant deux cent soixante et sept feuillets, cottés et paraphés, depuis le premier jusqu'au dernier par Nous Soussigné, l'un des Vicaires Généraux de Monseigneur Ignace Bourget, Evêque du dit Montréal le quinze Novembre Mil huit cent quarante.

Tome IV". - f. 1.

H. Hudon, V.G.

2. Texte des Actes. - f. 2-254 v.

3. "Table des Actes et pièces contenus dans le quatrième volume des Registres des Archives du Diocèse de Montréal". - f. 255-267 v.

RC 5 - 8 septembre 1843 - 5 août 1846 :

1. Titre: "Registre authentique des pièces et actes concernant le diocèse de Montréal, contenant deux cent quarante quatre feuillets cottés et paraphés depuis le premier jusqu'au dernier, par Nous Soussignés, l'un des Vicaires Généraux de Monseigneur Ignace Bourget, Evêque du dit Montréal, le quatre octobre, mil huit cent quarante-trois.

Tome V". - f. 1.

H. Hudon, V.G.

2. Texte des Actes. - f. 2 v. -234 v.

3. "Table alphabétique des matières contenues dans ce volume". - f. 235-244.

4. Carême: Tableau des Paroisses auxquelles les RR.PP. Oblats ont porté secours pendant le carême de 1846 . - f. 244 v. 
RC $6-22$ août $1846-16$ avril 1849 :

1. Titre: "Registre authentique des Pièces et Actes concernant le Diocèse de Montréal, contenant deux cent soixante quinze feuillets, cottés et paraphés depuis le premier jusqu'au dernier par nous soussigné, l'un des Vicaires Généraux de Monseigneur Ignace Bourget, Evêque du dit Montréal, le vingt deux août mil huit cent quarante six.

2. Texte des Actes. - f. 2-273 v.

H. Hudon, v.g. - VI". - f. 1.

3. "Table Alphabétique des Matières contenues dans ce Volume". - f. 274282.

RC 7 - 17 avril 1849 - 10 juillet 1852 :

1. Titre: "Ce cahier est substitué au Registre No 7 de la Chancellerie (17 avril 1849 - 10 juillet 1852) qui a été brulé lors de l'incendie de l'Evêché de Montréal en 1852. (cf. "Mandements, etc....", Vol. 8, p. 87: Postscriptum à la circulaire de Mgr Bourget du 3 septembre 1852.). - Il comprend seulement les ordinations publiées dans les "Mélanges Religieux" de cette époque.

Mgr G.-Robert Mitchell, P.D., Chancelier. Chancellerie de Montréal, le 29 mars 1956." - 1 p.

2. Table. -2 p.

3. Liste des ordinations. - p. 1-11.

4. Ordinations de prêtres repérées depuis 1956. - p. 12.

N.B. Tout ce registre est dactylographié.

RC 8 - 11 juillet 1852 - 27 février 1855 :

1. Titre: "Registre authentique des Pièces et Actes concernant le diocèse de Montréal contenant deux cents quarante-quatre feuillets, cottés et paraphés depuis le premier jusqu'au dernier par Nous soussigné, l'un des Vicaire Généraux de Monseigneur Ignace Bourget, Evêque du dit Montréal, le onze juillet mil huit cent cinquante-deux.

2. Textes des Actes. - f. 3 - 236 .

A. F. Truteau, Vic. Gén.”. - f. 1.

3. "Table Alphabétique des Matières contenues dans ce Registre". - f. 236 v. $-240 \mathrm{v}$.

RC 9 - 22 janvier 1855 - 3 octobre 1860 :

1. "Table Alphabétique des Matières contenues dans ce Volume". - 13 p. non numérotées.

2. Titre: "Régistre Authentique des Pièces et Actes concernant le Diocèse de Montréal, contenant deux cents vingt deux feuillets, cottés et paraphés depuis le premier jusqu'au dernier par Nous Soussigné, l'un des VicairesGénéraux de Monseigneur Ignace Bourget, Evêque du dit Diocèse de Montréal, le treize juin mil huit cent cinquante-cinq. - A.F.T. v.g.". f. 1 .

3. Texte des Actes. - f. $2-220$ v.

RC 10 - 7 octobre 1860 - 9 février 1873 :

1. Sans page-titre.

2. Texte des Actes. - f. 1-230.

3. Table alphabétique des matières. - 44 pages non numérotées. 
RC 11 - 22 février $1873-13$ mai 1880 :

1. Index alphabétique - Registre XI - 30 p. non numérotées.

2. Titre: "Registre authentique des Pièces et Actes qui concernent le Diocèse de Montréal, contenant deux cent cinquante feuillets, dont le premier et le dernier ont été cottés et paraphés par nous soussigné, Vicaire Général de Monseigneur Ignace Bourget, Evêque de Montréal. Montréal, le vingt un Février mil huit cent soixante-treize. -

3. Texte des Actes. - f. 2-250.

H. Moreau, Vic. Gén.". - f. 1.

4. Index des Chemins de Croix, Dispenses de vaux, Erection de confréries, Exhumations, Lettres Pastorales, Mandements Episcopaux, Bulles, Brefs et Rescrits Pontificaux, Obédiences. - 12 pages non numérotées.

\section{INVENTAIRES DE LA CORRESPONDANCE \\ DES ÉVÊQUES DE MONTRÉAL PARUS DANS LE RAPPORT DE L'ARCHIVISTE DE LA PROVINCE DE QUÉBEC}

\section{ABBE LOUIS-ADELLARD DESROSIERS :}

\section{Rapport 1941-1942}

Inventaire de la correspondance de Mgr J.-J. Lartigue - 1819-1826 incl., p. 345-496;

\section{Rapport 1942-1943}

Inventaire de la correspondance de Mgr J.-J. Lartigue (suite) - 1827-1832 incl., p. 1-174;

\section{Rapport 1943-1944}

Inventaire de la correspondance de Mgr J.-J. Lartigue (suite) - 1833-1835 incl., p. 209-334;

\section{Rapport 1944-1945}

Inventaire de la correspondance de Mgr J.-J. Lartigue (suite) - 1836-1837 incl., p. 173-266;

\section{Rapport 1945-1946}

Inventaire de la correspondance de Mgr J.-J. Lartigue (fin) - 1838-1840 incl., p. 39-136.

Inventaire de la correspondance de Mgr Ignace Bourget - 19 mai 1837 25 avril 1840 , p. 137-224;

\section{Rapport 1946-1947}

Inventaire de la correspondance de Mgr Ignace Bourget (suite) - 19 avril 1840-1841 incl., p. 81-175;

\section{Rapport 1948-1949}

Inventaire de la correspondance de Mgr Ignace Bourget (suite) - 1842-1843 incl., p. 343-477; 


\section{RÉVÉREND PÈRE LÉON POULIOT, s.j. :}

\section{Rapport 1955-1956 et 19561957}

Inventaire de la correspondance de Mgr Ignace Bourget (suite) - 1844, p. 179-221;

\section{Rapport 1961-1964 (Tome 42)}

Inventaire de la correspondance de Mgr Ignace Bourget (suite) - 1845, p. 9-68;

\section{Rapport 1965 (Tome 43)}

Inventaire de la correspondance de Mgr Ignace Bourget (suite) - 1846, p. 87-132;

\section{SÉRIE RD - REGISTRES DE DÉCRETS}

RD 1 - Registre de Décrets - Tome I - 1792 - 1841 :

1. Index. $-27 \mathrm{p}$.

2. Titre: "Registre authentique des pièces et actes qui concernent l'Erection des Paroisses, ainsi que les bâtisses ou réparations d'Eglises, Chapelles, Sacristies, Cimetières et Presbytères, dans le diocèse de Mont-réal du Bas-Canada; le dit registre contenant cent quatre-vingt feuillets, cottés et paraphés, depuis le premier jusqu'au dernier, par nous sousigné, vicaire général de [ces cinq derniers mots sont dans la marge] Mon-Seigneur J. J. Lartigue, premier Evêque de Mont-réal, au dit Mont-réal ou Ville-Marie, le premier janvier, Mil huit cent trente-sept. Cinq mots à la marge bons.

3. Texte des décrets. - f. 2-171.

Ig. Bourget Vic. Gén.”. - f. 1.

4. Tables des Actes et des Pièces contenus dans ce Volume. - f. 172-176 v.

5. Pages blanches. - f. 177-180 v.

RD 2 - Registre de Décrets - Tome II - 1841 - 1850 :

1. Texte des décrets. - f. 1-316 v.

2. Table. - f. 317-318.

RD 3 - Registre de Décrets - Tome III - 1850 - 1865 :

1. Titre: "Registre authentique des pièces et actes qui concernent l'érection des Paroisses ainsi que les bâtisses ou réparations d'Eglises, Chapelles, Sacristies, Cimetières et Presbytères, dans le Diocèse de Montréal, du Bas-Canada; le dit registre contenant trois cent vingt huit feuillets, cottés et paraphés, depuis le premier jusqu'au dernier, par Nous, soussigné, Vicaire Général de Monseigneur Ignace Bourget, Evêque du dit Montréal, le dix-huit Octobre mil huit cent cinquante.

2. Texte des décrets. - f. 2-327.

A. F. Truteau, Vic. Gén.". - f. 1.

3. Table chronologique des décrets intercalée entre les feuillets 127 et 128 . $-4 \mathrm{p}$.

4. Table des matières. - f. 328-329 v.

RD 4 - Registre de Décrets - Tome IV - 1865 - 1878 :

1. Titre: "Registre authentique des pièces et actes qui concernent l'érection canonique des paroisses, ainsi que les bâtisses ou réparations d'églises, 
Chapelles, Sacristies, Cimetières et presbytères, dans le Diocèse de Montréal, Bas-Canada; le dit registre contenant deux cents vingt-six feuillets, celui-ci y compris, cottés, paraphés, depuis le premier jusqu'au dernier, par Nous, A. F. T. Vicaire général de Mgr Ignace Bourget, Evêque Catholique du dit Montréal, le vingt-trois du mois de Mai, mil huit cent soixante et cinq". - f. 1 .

2. Texte des décrets. - f. 1 v - 221 v. [Avant 1877: f. 1 v. - 193 v.]

3. Table chronologique. - f. 221 v. -225.

4. Paroisses érigées par Monseigneur Bourget et démembrées de NotreDame. - 1 p. intercalée avant la page de garde de la fin du registre.

\section{SERIE RNLP - REGISTRES DE NOMINATIONS LETTRES DE POUVOIR}

\section{RNLP $1-7$ avril 1866 au 1er octobre 1878 :}

1. Cahier à onglets contenant 247 nominations numérotées 1 à 247. [Avant 1877: 1 à 238].

2. Index alphabétique des noms de prêtres. $-5 \mathrm{p}$.

\section{SERIE RCO - REGISTRES DE CONFIRMATIONS}

\section{RCO 1 - Registre de Confirmations - du 1er septembre 1861 au 8 octobre 1869 :}

"Noms des personnes confirmées en dehors des "grandes confirmations" faites à la Paroisse". - 45 p.

RCO 2 - Registre de Confirmations - du 8 novembre 1869 à décembre 1894 :

“Noms des personnes qui ont été confirmées dans la Cathédrale de Montréal et qq. autres endroits." - 182 p. [Avant 1877: 63 p.]

\section{SERIE RDM - REGISTRES DE DISPENSES DE MARIAGES}

RDM 1 - Registre de Dispenses de Mariages - 1822 - 1855 :

1. "Règlement du Conseil Souverain de Québec pour les curés et Seigneurs haut Justiciers de Canada." -2 p.

2. "Dissertation sur les cas Réservés écrite en 1784 par Frs. Jh. Deguise, diacre." $-84 \mathrm{p}$.

3. "Extrait de quelques clauses du Mandement de Monsg. l'Evêque de Québec - Le ler Novembre 1767." - 4 p.

4. "Extrait de Mr Buffon, Sur les Serins." -8 p.

5. "Manière d'élever les Jeunes Serins: méthode du baron Shaftalisque (?), officier allemand qui a élevé beaucoup de serins." - $2 \mathrm{p}$.

6. "Règlement que le Roi veut être observé au sujet de la concession des bancs dans les églises du Canada." -2 .

7. "Dispenses accordées depuis le 1er janvier 1822 par moi - Deguise, ptre, Vic. Gén." - 93 p. 
RDM 2 - Registre de Dispenses de Mariages - 1829 - 1837 :

1. "Registre de dispenses de mariages accordées par MonSeigneur l'Evêque de Telmesse depuis le 23 décembre 1829.

2. Liste des dispenses. - p. 1-196.

Ig. Bourget, Ptre, Secr."

3. Deux billets promissoires collés sur la $3 \mathrm{e}$ p. couverture, datés du 18 avril et du 14 juillet 1837 .

RDM 3 - Registre de Dispenses de Mariages - 1834 - 1840 :

1. "Tarif du Diocèse pour les componendes de dispenses." $-2 e$ p. couverture. -1 p.

2. "Registre des Dispenses pour Mariages accordées par nous, soussigné, Vicaire-Général du Diocèse de Québec depuis le dix mai 1834.

J. Bédard, ptre, v.g." - p. 1-40.

RDM 4 - Registre de Dispenses de Mariages - 1837 - 1848 :

1. "Régistre des Dispenses de Mariages accordées par Monseigneur l'Evêque de Montréal depuis le 27 septembre 1837.

2. Tarif des Componendes. -1 p.

A. F. Truteau, Ptre, Secrétaire." - 1 p.

3. Liste des dispenses. $-180 \mathrm{p}$.

RDM 5 - Registre de Dispenses de Mariages - 1841 - 1881 :

1. Texte du "serment exigé de la partie protestante". - $1 \mathrm{p}$.

2. Liste des tarifs de dispenses. $-1 \mathrm{p}$.

3. Liste des dispenses accordées par (nom pas indiqué), du 5 mai 1841 au 20 juin 1847. - $40 \mathrm{p}$.

4. Liste des dispenses accordées par M. Truteau, v.g., du 11 janvier 1848 au 28 novembre 1851 . $-18 \mathrm{p}$.

5. Liste des dispenses accordées par M. H. Moreau, v.g., du 26 décembre 1878 au 14 juin 1880 . -74 p.

6. Liste des dispenses accordées par M. N. Z. Lorrain, v.g., du 9 août 1880 au 9 juillet 1881 . - $55 \mathrm{p}$.

RDM 6 - Registre de Dispenses de Mariages - 1849 - 1851 :

1. "Registre de dispenses de mariages accordées par Monseigneur de Montréal depuis le vingt-un Octobre mil huit cent quarante huit jusqu'..." 1 p.

2. Liste des dispenses accordées par Mgr l'Evêque de Montréal du 21 octobre 1848 au 31 décembre 1851. - $92 \mathrm{p}$.

RDM 7 - Registre de Dispenses de Mariages - 1852 - 1858 :

1. "Registre des Dispenses de mariage accordées au Secrétariat de l'Evêché de Montréal depuis le cinq janvier mil huit cent cinquante-deux jusqu'au quinze février mil huit cent cinquante huit.

2. Liste des dispenses. $-245 \mathrm{p}$.

J. O. Paré Chan. secrétaire." - 1 p.

RDM 8 - Registre de Dispenses de Mariages - 1852 - 1871 :

1. "Registre des dispenses de mariage accordées par Mr le Vicaire Général de l'Evêché, depuis le (?) janvier mil huit cent cinquante deux jusqu'..." $-1 \mathrm{p}$. 
2. Liste des dispenses accordées par $M$. le Vicaire Général de l'Evêché du 19 janvier 1852 au 26 mai 1871 . - $276 \mathrm{p}$.

RDM 9 - Registre de Dispenses de Mariages - 1852 - 1880 :

1. "Registre des dispenses de Mariage accordées par Mr le Vicaire Général de Vaudreuil, depuis le 2 janvier mil huit cent cinquante deux jusqu'au..." et "Tarif des Componendes." - 1 p.

2. Liste des dispenses accordées par M. le Vicaire Général de Vaudreuil depuis le 2 janvier 1852 jusqu'au 14 février 1888 . - $94 \mathrm{p}$.

3. Liste des dispenses accordées et inscrites par Monseigneur E.-C. Fabre, Evêque de Montréal du 23 août 1873 au 27 décembre 1880. - 116 p. [Avant 1877: 52 p.]

4. 2 notes de Mgr E.-C. Fabre collées sur la 3e page couverture.

RDM 10 - Registre de Dispenses de Mariages - $1852-1864$ et 1880 - 1883 :

1. "Registre des Dispenses de mariage accordées par Mr Antoine Manseau, Vicaire Général, depuis le 23 janvier mil huit cent cinquante deux jusqu'au 20 octobre 1864 - et dispenses accordées par Mgr l'Evêque de Montréal et inscrites par T. Harel, ptre, Chancelier, du 11 mars 1880 au ..." - 1 p.

2. Liste des dispenses accordées par M. Antoine Manseau, Vicaire Général du 10 janvier 1852 au 20 octobre 1864. - 144 p.

3. Liste des dispenses accordées par Mgr E.-C. Fabre, Evêque de Montréal et inscrites par T. Harel, ptre, Chancelier, du 11 mars 1880 au 25 mai 1883. - $134 \mathrm{p}$.

3. 2 notes collées sur la $3 \mathrm{e}$ p. couverture.

RDM 11 - Registre de Dispenses de Mariages - 1858 - 1867 :

1. "Tarif des Componendes": collé sur la 2e p. couverture.

2. "Registre des Dispenses de Mariage accordées au Secrétariat de l'Evêché de Montréal depuis le vingt-six février mil huit cent cinquante-huit jusqu'au 14 novembre $1867 . "-1 \mathrm{p}$.

3. Liste des dispenses. $-330 \mathrm{p}$.

4. Note, collée sur la $3 \mathrm{e}$ p. couverture, sur la validité du baptême de la partie protestante, d'où s'ensuit la validité du mariage. - Autographe de Mgr Bourget.

RDM 12 - Registre de Dispenses de Mariages - 1871 - 1872 et 1873 - 1878 :

1. "Registre de dispenses de mariage accordées par le Vicaire Général de l'Evếché depuis le 27 mai mil huit cent soixante-et-onze au 21 décembre 1878." - 1 p.

2. Liste des dispenses accordées par M. A.-F. Truteau, du 27 mai 1871 au 25 novembre 1872 . $-68 \mathrm{p}$.

3. Liste des dispenses accordées par M. H. Moreau, du 24 février 1873 au 21 décembre 1878. - 268 p. [Avant 1877: 194 p.]

\section{SÉRIE RCC - REGISTRES DU CHAPITRE DE LA CATHÉERALE}

RCC 1 - 18 janvier 1841 au 15 janvier 1920 :

1. Titre: "Tabulæ publica in ducentis quinquaginta duobus foliis comprehensæe, ad Capituli Cathedralis B. Jacobi Apostoli Marianopolitani die XVIII 
Januarii M.D.CCCXLI erecti Acta authentica referenda Numero appositæ ac Subscripti Vicarii Generalis nominis propria nota insignitæ. Volume I - Marianopolitani - MDCCCXLI." - f. 1 .

2. Textes des Actes officiels de l'Evêque relatifs au Chapitre (Règlement, Nominations, etc.). - 18 janvier 1841 au 29 août 1908. - [Avant 1877: f. 2 à $116 \mathrm{v}$.] - f. $2-144 \mathrm{v}$.

3. Nominations du 4 novembre 1907 au 15 janvier 1920: 11 p. collées au registre entre le f. 144 et le f. 145.

4. Pages blanches. - f. 145-149.

5. Actes authentiques des Délibérations du Chapitre du 19 avril 1844 au 16 avril 1886. - [Avant 1877: f. 150 à 237 v.] - f. 150-247.

6. Après le f. 250: Actes authentiques des Délibérations du Chapitre du 10 octobre 1878 au 13 février 1882. - Numérotés, p. 4 à 73.

\section{SÉRIE RG - REGISTRES DU GÉSU}

RG 1 - 23 septembre 1852 au 11 août 1912 :

1. Années et pages correspondant à ces années. $-1 \mathrm{p}$.

2. "Names of adults baptized and received into the church, from the year 1852 to 1871 inclusively, in St Mary's College and the Church of Gesu, Montréal. This list is copied from two copy-books, which contain all the requisite information about those persons, and which are now in the Archives of St-Mary's College." et actes correspondant à ces années. p. 1 à 6.

3. Actes du 30 mai 1872 au 11 août 1912. [Avant 1877: p. 7 à 17] - p. 7 à 62.

RG 2 - 23 septembre 1852 au 10 janvier 1942 :

1. Lettre de l'abbé R. Mitchell, chancelier, au R.P. E. Cambron, s.j., recteur, Collège Ste-Marie, du 12 mai 1942, faisant part de la décision de l'Archevêque qu'il n'y ait plus de baptêmes ni de mariages célébrés dans la chapelle du Gésu et que les registres jusque-là utilisés soient envoyés aux Archives de l'Archevêché. - $1 \mathrm{p}$.

2. Actes des baptêmes et mariages célébrés dans la chapelle du Gésu du 23 septembre $1852 \mathrm{au} 10$ janvier 1942 . - $100 \mathrm{p}$.

\section{SÉRIE REC - REGISTRES DE L'ÉTAT CIVIL}

Normalement, les Archives diocésaines ne possèdent pas de registres de l'Etat civil dans la Province de Québec. Il peut arriver que, pour des raisons de sécurité ou de préservation, on dépose les registres de l'état civil d'une paroisse aux Archives d'un Evêché.

\section{SOUS-SÉRIE REC 1 - PAROISSE DE LA PURIFICATION - REPENTIGNY (1679 - 1827)}

Par tradition orale, nous avons appris que ces registres ont été remis aux Archives de la Chancellerie de l'Archevêché de Montréal par l'exécuteur testamentaire d'une personne qui les avait empruntés, de son vivant, soit pour les transcrire, soit pour y faire des recherches généalogiques. 
Ces registres sont dans un tel état de détérioration qu'ils ne sont pas accessibles aux chercheurs. On est prié de s'adresser à la paroisse qui en possède une transcription.

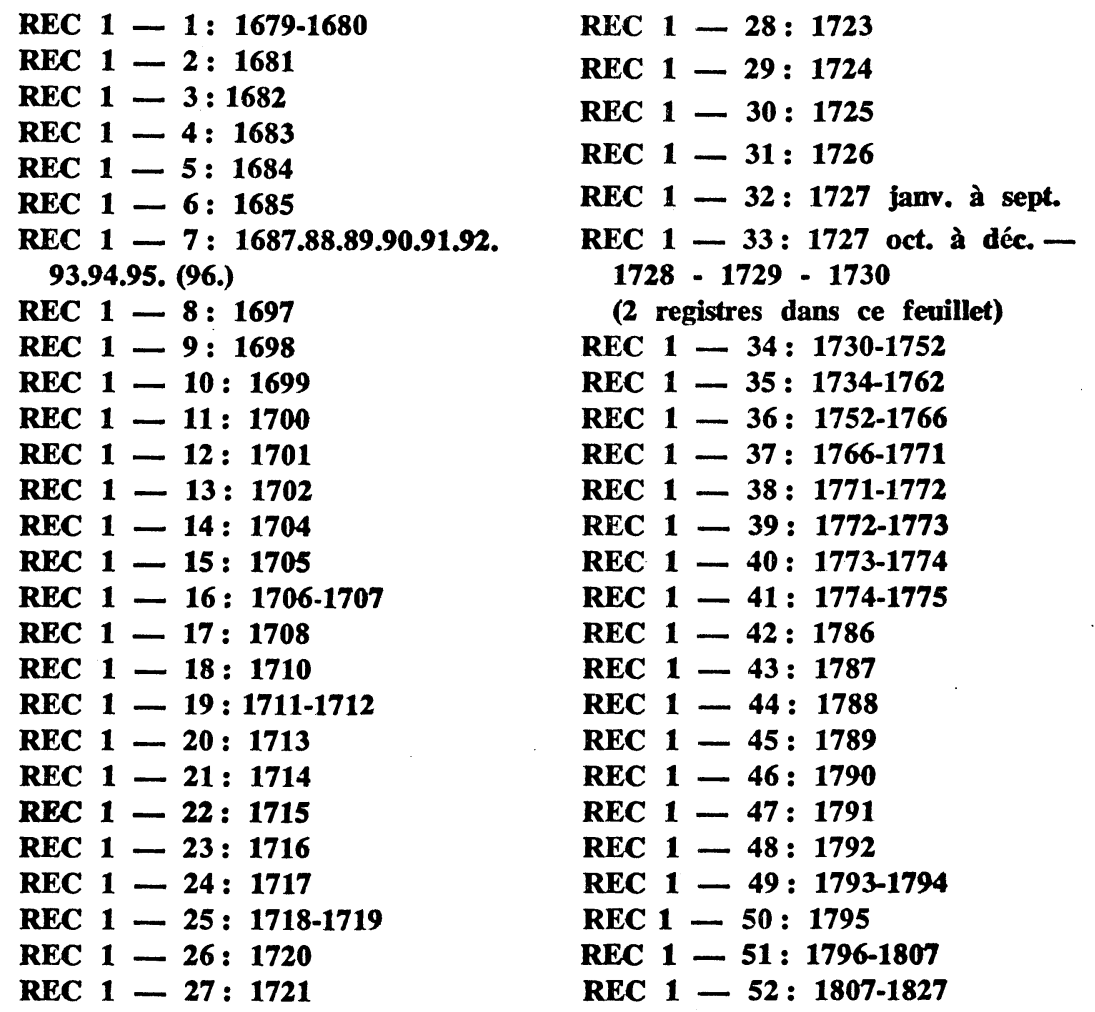

Note : Les registres suivants manquent : 1686; avril 1695 à avril 1697; 1703; 1709; 1712; 1722; août 1775 à janvier 1786.

\section{SÉRIE RCD - REGISTRES ET CAHIERS DIVERS}

Introduction. - Cette série comprend au-delà de 140 registres et cahiers divers. Tous ne sont pas mentionnés ici. Ceux qui ne sont pas mentionnés contiennent la totalité de leurs inscriptions au-delà de 1876.

On trouve dans cette série des originaux et des copies; des manuscrits et des imprimés; des documents officiels, officieux et privés. Enfin, le titre de cette série dit assez qu'on y peut trouver de tout. 
RCD 1 - Écrits de Mgr Bourget - T. 1 - 1840 - 1860 :

1. Titre: "Ecrits de Mgr Ig. Bourget - Fondateur des Filles de la Charité - Servantes des pauvres - Montréal - Volume I - 1840-1860". - 1 p.

2. Transcription des documents de Mgr Bourget relatifs aux Filles de la Charité Sours de la Providence - 19 décembre 1840 au 31 décembre 1859 - Scur Henri, copiste - p. 3 à 582.

3. Table des matières contenues dans ce volume. - - p. 583 à 588 .

RCD 2 - Ecrits de Mgr Bourget - T.2 - 1860 - 1870 :

1. Titre: idem, sauf "Volume II - 1860-1870". - 1 p.

2. Transcription de documents de Mgr Bourget relatifs aux Filles de la Charité Sours de la Providence - ler février 1860 au 3 décembre 1869 - Sceur Henri, Copiste. - p. 1 à 433.

3. Table des matières contenues dans ce volume. - p. 435 à 441 .

RCD 3 - Ecrits de Mgr Bourget - T. 3 - 1870 - 1880 :

1. Titre: idem, sauf "Volume III - 1870-1880". - 1 p.

2. Transcription de documents de Mgr Bourget relatifs aux Filles de la Charité Sœurs de la Providence - 8 avril 1870 au 25 décembre 1879 Sour Henri, copiste. - p. 1 à 618. [Avant 1877: p. 1 à 190].

3. Table des matières contenues dans ce volume. - p. 621 à 629 .

RCD 5 - Lettres de Jacques Viger :

Correspondance entre J. Viger et le Major Codd et autres - Autograpbe 22 novembre 1821 au 5 mai 1827 . - 146 p.

RCD 6 a 13 - Journal du Voyage de Mgr Joseph-Octave Plessis en Europe 1819 à 1820 :

Multiples transcriptions de parties de ce journal de voyage qui a paru en 1903: Journal d'un voyage en Europe par Mgr Joseph-Octave Plessis, Québec, Imprimerie S. A. Demers, 1903. 469 p.

RCD 14 - Mgr Bourget - Registre pour les Ordonnances de Visites pasto. rales - 1861 à 1863 :

1. Observations générales sur les exercices de la Visite Pastorale. - f. 1 à 4 .

2. Ordonnances du 3 juin 1861 au 19 octobre 1861. - f. 5 à 32.

3. Itinéraire de la Visite Pastorale de 1861 . - f. 32 et $32 \mathrm{v}$.

4. Remarques sur la Visite. - f. 33 et $33 \mathrm{v}$.

5. Visite pastorale de 1863 . - du 10 au 28 juin. - f. 34 à 39.

6. Liste des paroisses. - f. 40 à 45 .

7. Pages blanches. - f. 45 v. à 126.

RCD 15 - Mgr E.-C. Fabre - Registre pour les ordonnances de la visite pastorale - 1873 - 1876 :

1. Titre : "Registre pour les ordonnances de la Visite Pastorale, contenant quatre-vingt seize feuillets celui-ci y compris. - Joseph Charette Acol.”. f. 1 .

2. Nom des paroisses et références aux pages. $-\mathrm{f} .1 \mathrm{v}$. 
3. Ordonnances de la Visite Pastorale faite par Mgr E.-C. Fabre du 11 juin 1873 au 11 juillet 1876 . - f. 2 à 96 .

4. Suite des Noms des paroisses et références aux pages. - en $3 e$ page couverture.

RCD 16 - Mgr E.-C. Fabre - Registre pour les ordonnances de la visite pastorale - 1876 - 1883 :

1. Titre: "Registre pour les Ordonnances de Visites Pastorales." - f. 1.

2. Noms des paroisses et références aux pages. - f. 1 et $1 \mathrm{v}$.

3. Ordonnances de la Visite Pastorale faite par Mgr E.-C. Fabre du 6 septembre 1876 au 23 juin 1883. - [Avant 1877: f. 2 à 67] - f. 2 à $87 \mathrm{v}$.

RCD 26 - Mgr Bourget - Journal des Affaires courantes du Diocèse de Montréal - 1844 - 1845 :

1. Titre: "Registre pour servir à l'entrée des affaires courantes du Diocèse de Montréal, à mesure qu'elles se présentent, pour être expédiées en tems convenable, commencé le 18 Décembre 1844 , et terminé en Décembre 1845; contenant vingt-sept feuillets, le dernier n'étant que commencé." - 1 p.

2. Après la page-titre, deux documents intercalés:

a - Lettre de l'abbé A. Doyle, curé de St-Anthony's, Whitehale, Wash. Co., N.Y. à Mgr Ig. Bourget, le 19 mai 1845 .

b - Lettre du P. J. Guerdet, à Mgr Ig. Bourget, envoyée de Salina, le 5 octobre 1846 .

3. Entrée des affaires courantes du Diocèse de Montréal. - 18 décembre 1844 à décembre 1845 . - p. 1 à 27.

4. Pièces intercalées:

a - Entre la p. 4 et 5: Lettre de John Murdoch à Mgr Ig. Bourget, adressée de Glasgow, le 24 mai 1845.

b-Entre la p. 10 et 11: Lettre de R. Robert, ptre, à Mgr Ig. Bourget. adressée de St-Polycarpe, le 29 août 1845 .

c-Entre la p. 12 et 13: Lettre de G. Chabot, ptre, à Mgr Ig. Bourget, adressée de St-Lin, le 28 août 1845 .

d-Collée à la p. 16: Lettre du P. P.-A. Telmon, o.m.i., à Mgr Ig. Bourget, adressée de Bytown, le $1^{\text {er }}$ septembre 1845.

e-Collée sous la précédente: Lettre de l'abbé John Stackett, à Mgr Ig. Bourget, adressée de Verplanks Point, N. Y., le $1^{\text {er }}$ juin 1845.

f-Collée à la p. 17: Lettre de M. Morin, ptre, à Mgr Ig. Bourget, adressée de Montréal, le 2 octobre 1845.

g-Collée à la p. 23: Lettre de J.-B. Marcotte, ptre, à Mgr Ig. Bourget, adressée de l'Ile Dupads, le 16 juin 1845.

h-Collée à la p. 26: Lettre du P. Jos. Tellier, s.j., à Mgr Ig. Bourget, adressée de Laprairie, le 20 septembre 1845.

\section{RCD 27 - Mgr Bourget - Journal des Affaires du Secrétariat de Montréal depuis le ler mai 1843 :}

1. Journal des affaires de l'Evêché du $1^{\text {ex }}$ mai 1843 au 31 décembre 1845 . $178 \mathrm{p}$. 
2. Pièces intercalées:

a - Au 28 mai 1845: Registers not returned.

b- Au 18 juillet 1845: Lettre de B. Ricard, ptre, à Mgr Ig. Bourget, adressée de Lacadie, le 14 juillet 1845 .

c-Sous la précédente: Lettre de B. Larocque, médecin, adressée de Lacadie, le 14 juillet 1845 .

d-Au 30 juillet 1845: Lettre de Thomas Cooke, ptre, à Mgr Ig. Bourget, adressée de la cure des Trois-Rivières, le 10 août 1845 .

e-Au ler août 1845: Lettre à M. H. Hudon, v.g., adressée de Lachine, le 28 juillet 1845 .

f - Au 24 décembre 1845: Lettre de Lebourdais, ptre, à Mgr Ig. Bourget, adressée de Rivière-du-Loup, le 8 janvier 1846.

RCD 28 - Mgr Bourget - Journal des Affaires courantes du Diocèse de Montréal à mesure qu'elles sont expédiées :

1. Du 4 janvier 1846 au 26 mai 1849. - p. 1 à 285.

2. Du 20 février 1854 au 29 mars 1854. - p. 286 à 292.

3. En retournant le registre dans l'autre sens, on trouve: "Entrée des Affaires courantes à mesure qu'elles se présentent, pour être ensuite expédiées en temps convenable depuis le 1er janvier 1846 jusqu' . . " - Du ler janvier 1846 au 18 août $1846 ; 9$ juin $1847 ; 6$ décembre et 22 décembre 1848; 25 avril au 31 décembre 1849; 1er au 5 janvier, 26 au 29 avril 1850 ; 22 juillet au 13 août $1851 ; 21$ mai au 24 mai $1852 ; 13$ mars au 29 mars, ler au 8 août 1854 . - p. 1 à 61.

RCD 29 - E.-C. Fabre - Cahier de notes - Études à Paris (mars 1843 octobre 1844) jusqu'à son entrée au séminaire d'Issy (octobre 44):

1. En page de garde, notes du Chanoine Emile Chartier, de l'Archevêché de Montréal, 28 mars 1932: "Notes rédigées à Paris par Edouard-Charles Fabre, le futur archevêque de Montréal, en 1843 et 1844 . Il a quitté le séminaire de St-Hyacinthe le 7 janvier 1843, s'est embarqué pour New-York le $1^{\text {er }}$ février, est monté sur la Duchesse d'Orléans le 11 février et est arrivé au Havre le 8 mars avec son père et $M$. Toupin. Le $1^{\text {er }}$ novembre 1843 , il note qu'il a fait sa $1^{\text {ère }}$ communion il y a six ans (1837) et, le 28 février 1844, qu'il a 17 ans et sa sceur Hortense (plus tard Lady Georges Cartier) 16. Le journal finit avec son entrée au séminaire d'Issy en octobre 1844".

2. En retournant le cahier dans l'autre sens, on trouve un relevé de revenus et dépenses du même. -29 p.

3. Intercalé en $3^{\text {e }}$ page couverture, un petit cahier de 14 feuillets, donnant un autre journal de voyage du 3 mai au 27 juin 1846 .

4. Intercalée au même endroit, une "liste des Elèves du Séminaire de St-Hyacinthe". -4 p.

RCD 30 - Société d'une Messe - Vol. I:

1. Bref historique de l'Association de Prières pour les Prêtres défunts. 2 p.

2. Circulaire de M. J.-O. Paré, ptre, datée du 26 juin 1862, à propos de la Société. - Imprimé - 2 p.

3. Section de trois messes - p. 1 à 3.

4. Section de une messe - p. 4 à la fin, par ordre alphabétique. 
5. Intercalé en la $3^{e}$ p. couverture: Liste des Prêtres de l'Archidiocèse de Québec, et des Diocèses de Trois-Rivières, St-Hyacinthe, Rimouski et de Sherbrooke, appartenant à la "Section Provinciale" de la Société d'une Messe. - 9 p.

6. Nécrologie des Membres de la Section d'une messe. - Du 20 janvier 1881 au 13 octobre 1888 . -4 p.

RCD 31 - Registre d'ordinations - 1873 - 1885 :

1. Consistoire du 21 mars 1873 - Liste des membres - 1 p.

2. Consécration de Mgr E.-C. Fabre - 1 ${ }^{\text {er }}$ mai 1873 - Evêques présents sur la même page.

3. Ordinations faites par Mgr E.-C. Fabre, év. de Gratianopolis - 11 mai 1873 au 30 août 1885 .

RCD 37 - Recueil d'Ordonnances et de Consultations canoniques :

1. Extraits des Ordonnances du Diocèse de Québec. - (1664-1700) p. 1-37.

2. 4 p. blanches.

3. "Questions soumises au Père Rosaven par Mrs Perreault et $\mathrm{Ch}$. Duchesney, pendant leur séjour à Rome, le 24 mai 1837." - 2 p. non numérotées.

4. "Recueil de réponses faites par Monsgr. l'Ev. de Québec et Mr Roux vic. gén. aux consultations particulières de quelques prêtres du Diocèse de Québec, depuis 1814." - 78 p. non numérotées.

5. 1 p. blanche.

6. "Extrait d'une lettre de l'Evêque de Québec à Son Eminence le Cardinal Antonelli, Préfet de la Sacré Congrégation de la Propagande, en date du 26 oct. 1792." - 6 p. non numérotées.

7. Lettre encyclique de Benoit XIV, $1^{\text {er }}$ novembre 1745, sur l'usure. $11 \mathrm{p}$. non numérotées.

8. "Opinion de Monsgr Troy, Archevêque de Dublin, touchant le prêt à intérêt, extrait traduit d'une lettre de ce prélat du mois de mai 1811, à Mgr Plessis, Ev. de Québec." - 15 p. non numérotées.

9. En retournant le cahier dans l'autre sens: "Acte ou ordonnance qui concernent la construction ou la réparation des églises, presbitères et cimetières (dans le diocèse de Québec) passé 30 avril 1791." — 5 p. non numérotées.

RCD 38 - Essai de Tarif, avec instructions sur le mariage, de Mgr J.-O. Plessis, Ev. de Québec:

Ex-libris sur la couverture: "Jean Olivier Chevrefils, Prêtre, curé de StConstant. No 725." - 25 p.

RCD 39 - Recueil de Mandements des Évêques de Québec sur les Fêtes (1767 - 1827) :

48 p. imprimées et 38 p. manuscrites.

RCD 40 - Mandements et Ordonnances des Évêques de Québec et de Montréal, 1660 - 1840 :

Collection de mandements imprimés ou transcrits dans ce registre de $360 \mathrm{p}$. ayant appartenu à M. F.-X. Marcoux, ptre; précédé d'une table des matières de $15 \mathrm{p}$. 
RCD 41 - Mission de Mgr Desautels à Rome - I (1872) :

1. Sur la couverture: "Questions principales - Démembrement de N. D. Coadjuteur - Université - Chapitre de Montréal - Ecoles du N. Brunswick - Code des Curés du Juge Beaudry - Pagnuelo - Etudes historiques - Fabriques - Grand St-Ours - Notre-Dame de Grâce Noces d'or de Mgr Bourget - Code civil - Index au IV cahier page 183." - Ce IVe cahier est présenté sous la cote RCD 44 de cet inventaire.

2. Pagination à partir de la face: p. 1-124. - Titré: "Documents relatifs à la Mission de Mgr Desautels à Rome en 1872." - 11 mai 1872 au 10 février 1873.

3. Pagination à partir de l'envers : p. 1-47. - Titré: "Canevas ou Brouillons de lettres écrites par Mgr Desautels en 1872." - 14 mai 1872 au 15 août 1872 .

RCD 42 - Mgr Desautels à Rome - I (1872 - 1873) :

1. Sur la couverture: "Questions principales - Université - Coadjuteur Chapitre de Montréal - Archevêque de Québec - Démembrement de N. Dame - Hôtel-Dieu - Grand St-Ours - Code civil - Elections politiques - Programme catholique - Ecoles du Nouveau-Brunswick Registre de l'Etat-civil - Journaux - Ecoles mixtes - A. Villeneuve Comédie infernale - Lettres diverses - Index au IVe cahier page 183." - Ce IVe cabier est présenté sous la cote RCD 44 de cet inventaire.

2. Pagination à partir de la face: p. 1-154. - 29 novembre 1872 au 29 août 1873.

3. Pagination à partir de l'envers: p. 1-18. - Brouillons de letires du 10 au 28 mars 1873.

RCD 43 - Mgr Desautels à Rome - III (1873) :

1. Sur la couverture: "Questions principales - Démembrement de N. Dame - Notre-Dame de Grâce - Cimetière de N. Dame de Montréal Université - Paroisse de St-Patrice - Bill des Paroisses - Code des Curés de Mr Beaudry - Fabrique de St-Angèle (3 Riv.) - Propriété d'une Eglise consacrée. - Index au IVe cahier page 183." - Ce IVe cahier est présenté sous la cote RCD 44 de cet inventaire.

2. Titre: "6e voyage à Rome" - p. 1.

3. Documents du 28 octobre 1873 au 18 mars 1874. - p. 1-117.

\section{RCD 44 - Mgr Desautels à Rome - IV (1873 - 1874) :}

1. En page du faux titre: "N.B. La majeure partie de la correspondance de Mgr Desautels - de Rome au Canada - se trouve au Cahier de Ste-Anne-de-Varennes - dont Mgr D. était Curé." - Ce cahier à onglets est maintenant aux Archives de la Chancellerie de l'Evêché de St-Jeande-Québec, à qui il a été remis lors de l'érection de ce diocèse en 1933.

2. En page-titre: "Questions principales - Grand St-Ours - Mr T. Maréchal - Biens des Jésuites - Parts de banques - Sociétés de Construction Démembrement de N. Dame de M. - Consultation de Mr Archambault, Curé de St-Timothée - D'Orsonnens (Gustave d'Odet) - Bulle de Clément XIV supprimant la Comp. de Jésus - Université - $\mathrm{La} \mathrm{Co}$ médie Infernale - A. Villeneuve - C. Vincelette - Programme catholique - Routhier (Juge) - Cause Drouin contre Rev. Mr U. Archambault - Index page 183."

3. Titre: "6e voyage à Rome. 1873-1874. Documents." 
4. Documents du 10 février 1874 au 25 novembre 1877. - p. 1 - 182 [Avant 1877: p. 1-175].

5. Index des quatre cahiers - p. 183-195. - Il s'agit des cahiers RCD 41, 42,43 et 44 .

RCD 45 - T. Harel, ptre - Lettres - I (22 août 1873 au 21 mai 1876) :

Ce cahier à onglets et le suivant (RCD 46) sont une collection des documents et de la correspondance envoyés ou reçus par $M$. Harel. Tout est divisé en sections, numérotées 2 à 17 , de façon continue à travers les deux cahiers; la pagination, elle aussi, est continue, d'une section à l'autre et d'un cahier à l'autre.

1. Sur la page de garde: "T. Harel, ptre - Correspondance et documents Rome - de Août 1873 à son retour au Canada en 1877. - Mémoires et notes écrites au Canada - octobre 1877."

2. Section 2: "Lettres de Mgr Bourget à T. Harel, ptre. - La Table des matières est à la fin du 2nd volume. - 2." - Du 22 aout 1873 au 16 mai 1877. - p. 1 à 229. [Avant 8 sept. 1876: p. 1-215.]

3. Section 3: "Lettres $1^{\circ}$ de Mgr Fabre $2^{\circ}$ de Mgr Pinsonnault, Evêque de Birtha à T. Harel, ptre. - 3."

$1^{\circ}$ Lettres de Mgr E.-C. Fabre, du 29 octobre 1873 au 29 mars 1877. p. 230-295. [Âvant 8 sept. 1876: p. 230-275.]

$2 \circ$ Lettres de Mgr Pinsonnault, du 30 avril 1874 au 26 mai 1875. p. 296-304.

4. Section 4: "1874 - Correspondance - 17 juillet 74 à 25 octobre (plus bas) - Note - Jusqu'au départ de Mgr Desautels de Rome au commencement de Juillet 1874 , le soussigné n'a pas gardé copie de ses lettres à Mgr Bourget, etc., parce qu'elles ne concernaient pas les affaires du Diocèse. - Le soussigné arrivé à Rome en septembre 1873, en est reparti en juin 1877 - Evêché de Montréal - 7 décembre 1885 T. Harel, Ptre, Chancelier - 4." Correspondance envoyée - p. 305-326.

5. Section 5: "1874 - Correspondance - 3 Décembre 74 à 22 février 1875 - 5." - Correspondance envoyée du 3 décembre 1874 au 28 (sic) février 1875. - p. 327-344.

6. Section 6: " 1875 - Correspondance - 11 avril 1875 à 26 octobre 1875 6." - Correspondance envoyée - p. 345-400.

7. Section 7: " 1875 - Correspondance - 1 novembre au 31 Décembre 7." - Correspondance envoyée - p. 401-447.

8. Section 8: "1876 - Correspondance - Janvier 11 jusqu’à 21 mai 1876 - 8." - Correspondance envoyée - p. 448-535.

RCD 46 - T. Harel, ptre - Lettres - II (22 mai 1876 au 22 août 1877) :

Voir note au début du cahier coté RCD 45.

9. Section 9: “Correspondance - Du 28 mai 1876 au 26 novembre 1876. 9." - Correspondance envoyée - p. 536-591.

10. Section 10: "Correspondance - De janvier 1877 au 19 mai 1881. (plus bas) Note - La dernière lettre adressée de Rome est du mois d'avril 1877 page - 627 - à la suite viennent certains mémoires du soussigné faits à Montréal sur les événements, au jour le jour. - $T$. Harel, Ptre Chancelier. - 10." - Une lettre reçue de l'abbé Louis Leduc, datée de Montréal le 15 décembre 1876; correspondance du 15 janvier 1877 au 22 avril 1877; mémoire du 21 octobre 1877, 23 octobre 1877, 30 octobre 1877, 2 lettres du 26 février 1878, mémoire 
du 2 octobre 1878, 4 mai 1881, 6 mai 1881, 7 mai 1881, 10 mai 1881, 19 mai 1881. - p. 592-660.

11. Section 11: "28 sept: 1874 - quelques remarques sur le $1^{\text {er }}$ argument dans le jugement rendu par S. Honneur le Juge Routhier dans la cause de Drouin vs M. Archambault, Curé. - 11." - La page-titre de cette section est numérotée: 661 . - p. 662-693.

12. Section 12: "Documents - 17 juillet 1874 à 8 janvier 1875 - 12." p. 694-726.

13. Section 13: "Documents - 27 janvier 1875 à octobre 1875 - 13." Documents du 10 janvier 1875 au 29 octobre 1875. - p. 727-763.

14. - Section 14: "Documents - Avril 1875 à Février 1876 - 14." Documents du 23 avril 1875 au 24 janvier 1876. - p. 764-821.

15. Section 15: "Documents - Février 1876 à (sic) - 15." - Documents du $1^{\text {er }}$ février au 20 octobre 1876 - p. 822-850.

16. Section 16: "A Son Em. le Card Oreglia de Mgr Laflèche - Septembre 1876. - 16." - Ce document et d'autres se trouvent aux pages 851-905.

17. Section 17: “Ste-Brigide - 10 novembre 1876 - 17." - p. 905 (2e page numérotée ainsi) - 926 .

18. Après la p. 926, Table des matières des cahiers RCD 45 et 46 . -21 p. non numérotées.

RCD 47 - Chan. P. Leblanc - Lettres - (1867 - 1881) :

Ce registre de 606 p. numérotées contient, de la p. 1 à la p. 262, la copie de la correspondance envoyée par le Chan. P. Leblanc du 27 mars 1867 au 26 mars 1881. Le reste en pages blanches, sauf à la page 606, copie d'une lettre du 27 janvier 1871. [Avant 1877: p. 1-235 et 606.]

RCD 48 - Offrandes pour la reconstruction de la Cathédrale - $(1870$ - 1877):

1. Coupures de journaux p. 1-9 et 17-38.

2. Manuscrit: "Noces d'or de Mgr Bourget Evêque de Montréal - 30 novembre 1872 - Offrandes faites à cette occasion (Noms et montant)." p. 11-16.

3. Avant les pages de garde de la fin du registre: "Noms des Députés des Paroisses pour Dîner." - 4 p. non numérotées.

RCD 49 - Dépenses de la Cathédrale - (1870 - 1880):

Dépenses du 18 mars 1870 au 23 mars 1880 . -23 p.

RCD 51 - Relations du Diocèse de Montréal à la S. C. de la Propagation de la Foi - (1855 - 1888):

Rapports faits à la Congrégation sur l'etat du Diocèse à l'occasion des visites "ad limina".

1. 29 juin 1855 - p. 2-17.

2. Avril 1862 - p. $18-40$.

3. Renseignements sur le Diocèse de Montréal pour l'information du StOffice - 13 mai 1869 - p. 41 bis.

4. Questionnaire pour les diocèses de Missions envoyé par ordre de Léon XIII. - imprimé - collé à la p. 42.

5. Accusé de réception du rapport suivant - 15 Décembre 1879 - imprimé - collé après la p. 43. 
6. "Rapport de Monseigneur E. C. Fabre, Evêque de Montréal à la S. Congrégation de la Propagande, dans sa visite ad limina apostolorum (1879-1880)."

7. "Rapport de Mgr Fabre, archevêque de Montréal concernant son archidiocèse. - 6 novembre 1888." - p. 44 [document de 22 p., paginées 1 à 21 , la dernière n'étant pas numérotée et la première portant la numérotation de la p. 44 du registre.]

RCD 73 à 83 - Abbé L.-A. Desrosiers - Cahiers manuscrits :

Ces cahiers contiennent la presque totalité du texte des inventaires de $\mathrm{Mgr}$ Lartigue et Bourget parus dans le Rapport de l'Archiviste du Québec sous la plume de l'abbé Desrosiers. Nous ne notons ici que les parties de cahiers contenant des notes qui n'ont pas paru dans les inventaires.

RCD 73 - Contient une liste du clergé 1821 - 1831;

une liste des Sulpiciens 1821 - 1931;

une liste des onze sulpiciens de 1794.

RCD 78 - Contient, à la fin, une liste des curés et vicaires en 1830.

RCD 79 - Contient, à la fin, une liste du clergé en 1833.

RCD 81 - Contient, au début, des notes de tous genres.

RCD 84 - Liste chronologique des Evêques et des Prêtres du Canada depuis l'établissement de ce pays :

Imprimé: "Liste chronologique / des évêques / et / des prêtres / tant séculiers que réguliers, employés au service de l'Eglise / du Canada / depuis l'établissement de ce pays / et aussi / la liste des évêques des autres possessions britaniques / de / l'Amérique du Nord. - / Revue au Secrétariat de l'Evêché de Québec. - / A Québec: / Chez T. Cary et Cie. Imprimeurs Libraires / au Chien d'Or, rue Buade. - / 1834." - XII - 52 p.

Ce volume est revu, complété et augmenté jusqu'en 1863 incl.

RCD 85 - Liste des Prélats et Ecclésiastiques décédés en Canada, Diocèse de Montréal :

1. Encyclique de Léon XIII - 4 août 1879 - p. 1-5.

2. Liste par Diocèses et par paroisses - Diocèses mentionnés: Montréal, Québec, Trois-Rivières, St-Hyacinthe, Bytown (Ottawa), Kingston, Toronto, St-Boniface et Sandwich (London) - (1642-1881) - p. 6-193, (sauf p. $140-145$ et 164-165: Liste de Prélats et de Prêtres morts et enterrés en différents lieux. - 1661, 1810-1881).

RCD 86 - Nécrologe des Prêtres tant séculiers que réguliers qui ont desservi le Diocèse de Montréal et qui sont décédés dans les anciennes limites de la Nouvelle-France :

La page titre indique avant ce titre: "1ère Partie" et après: "Janvier à Juin compris". Ce travail est un autographe de Jacques Viger. Classées selon les mois et les dates de décès des prêtres, les inscriptions donnent, pour chacun, le lieu du décès, la date, l'âge, les localités desservies par le prêtre durant sa vie, et des remarques. - (1675-1851) - 56 p. 
RCD 87 - Titres de Missions - (1822 - 1889) :

1. Cahier à onglets contenant 393 documents par la signature desquels les ecclésiastiques, avant le sous-diaconat, s'engageaient à travailler au service du diocèse, et non pas dans une communauté religieuse. Les deux premiers sont de Mgr I.artigue et Mgr Bourget.

2. Index alphabétique $-24 \mathrm{p}$.

\section{RCD 88 - Registre Lennox :}

Cahier de notes de 92 p. de John Manner Kerr Lennox (1802-1832), fils issu du ler mariage de l'épouse de Jacques Viger.

1. Sous la couverture du titre: Titres de noblesse et généalogie (1384-1802). $-16 \mathrm{p}$.

2. En retournant le registre dans l'autre sens: Correspondance écbangée au sujet d'un héritage de son père. - $13 \mathrm{p}$.

\section{RCD 93 - Historique de la Cathédrale :}

Coupures d'un album appelé Le Bazar publié à la fin du siècle dernier pour susciter la générosité des fidèles en faveur de la reconstruction de la Cathédrale de Montréal. - $51 \mathrm{p}$.

RCD 94 - H. Moreau, ptre : Récit de voyage en mission en 1840 -

H. Moreau, ptre, vécut de 1815 à 1880 . Missionnaire dans la vallée du haut Ottawa, Témiscamingue et Abitibi (1839-1843), il fut curé (1843-1853), puis termina sa vie sacerdotale à l'Archevêché de Montréal (1853-1880), où il fut archidiacre, et enfin vicaire général du diocèse de Montréal (1873-1880). Cahier de $83 \mathrm{p}$.

1. Récit de voyage en mission au Témiscaningue. -26 maj au 15 septembre 1840.

2. En retournant le cahier dans l'autre sens: quelques notes, à la mine.

RCD 95 - H. Moreau, ptre : Cahier de notes et de correspondance -

1. "Pour l'ouverture d'une mission chez les Infidèles" - En langue indienne. - (Voici les premiers mots du texte: "Enicinabéwiieg nainawisiwiieg ki pi tipadjimottonim ...") - p. 1-11.

2. p. 12 - blanche.

3. Notes de prédication sur divers thèmes. - p. 13-50.

4. "Lettres envoyées." - (1845-1853) - p. 51-118.

5. "Programme des Socialistes/ de tous les temps/ de toutes/ les couleurs/ Rouges/ Liberté, Egalité, Fraternité." - p. 119.

6. En retournant le cahier dans l'autre sens: "Titres que le baptême conferre à celui qui le reçoit." - p. 1 .

7. Notes en langue indienne, à la mine, presque effacées. - p. 2-4.

8. Texte de son premier sermon comme curé. - p. 5-7.

9. p. 8-22 - blanches.

10. Notes de prédication - p. 23-68.

11. Lettre du 23 décembre 1852 au Dr Dumouchelle. - p. 69.

RCD 96 - Frs. Porlier, ptre - Terrebonne - Cahier de notes (? - 1865 ?) : François-Pascal Porlier (1802-1869) fut curé à Terrebonne, St-Philippe, Blairfindie, Les Cèdres, La Pointe-aux-Trembles. 
1. Notes généalogiques de sa famille. $-7 \mathrm{p}$.

2. Voyage en Europe de Mgr Plessis pour la période du 30 octobre 1819 au 1er mai 1820 . - $266 \mathrm{p}$.

3. Biographie de quelques membres du Clergé du Canada. - 57 p. (Date ultime établie d'après une référence à un article paru en 1865 , mentionné dans une des biographies).

RCD 97 - A. Piché, ptre - Lachine (1869) : Petit cahier de notes de voyage Notes de voyage. - Vol. 1 - Sites à visiter en France, à Rome, à Jérusalem. $-13 \mathrm{p}$.

RCD 98 - A. Piché, ptre - Lachine (1869) : Notes de voyage - Vol. 2 Notes de voyage. - Vol. 2 - Rome et Jérusalem. - 8 février au 31 mars $1869-164 \mathrm{p}$.

RCD 99 - A. Piché, ptre - Lachine (1868) : Notes de voyage - Vol. 3 Notes de voyage. - Vol. 3 - Marseille à Rome. - 4 décembre 1868 au 7 février $1869-122 \mathrm{p}$.

RCD 100 - A. Piché, ptre - Lachine (1869) : Notes de voyage - Vol. 4 Notes de voyage. - Vol. 4 - Jérusalem, Rome, Pise, Bologne, Venise, Milan, Turin. - 31 mars au 28 juin 1869 . - $147 \mathrm{p}$.

RCD 101 - A. Piché, ptre - Lachine (1869): Notes de voyage - Vol. 5 Notes de voyage - Vol. 5 - Suisse, Allemagne, Belgique, France et Canada. -28 juin au 31 août 1869 . - 204 p.

RCD 106 - Mgr Bourget - 2e voyage en Europe - 29 septembre 1846 au 24 avril 1847 :

Presque tout est de la main de Mgr Bourget.

1. Correspondance et mémoires - 30 septembre 1846 au 11 février 1847 p. 1-24.

2. Index - page après la p. 24 et une autre page marquée "a".

3. Après plusieurs pages blanches: correspondance du 11 janvier 1847 au 24 avril 1847. - p. 24-111.

4. En retournant le registre dans l'autre sens: "Journal du 2 e voyage de l'Ev. de Montréal en Europe." - en marge: "A partir du 29 septembre 1846 au 4 décembre de la même année." - p. 1-54. (cf. $R A P Q 1965,89$.

RCD 107 - Mgr Bourget - Rome, 18 - 12 - 64 à 13 - 6 - 65 :

Correspondance datée de Rome, lors du voyage en Europe de 1864-1865.

1. 18 décembre 1864 au 31 mai 1865. - p. 16-236.

2. 1er juin 1865 au 13 juin 1865 . - p. 1-15.

3. Index des lettres -8 p. intercalées après la p. 236.

La correspondance est écrite soit par Mgr Bourget soit par un copiste. L'index est de M. T. Harel, ptre, Chancelier.

RCD 109 - Transcription dactylographiée de la Relation du voyage de l'Évêque de Montréal en Europe en 1841 :

Original de la dactylographie du manuscrit conservé en RLL 9, p. 287-559. (cf. $R A P Q 1965,89$.) 
RCD 110 - idem : Copie de la transcription.

RCD 111 - Registre des inhumations dans la crypte de la Cathédrale (1840 1854) :

22 Actes d'inhumation $-12 \mathrm{p}$.

RCD 112 - Journal du voyage de Mgr Fabre - (1869 - 1870) :

1. Liste des correspondants à qui des lettres ont été envoyées par Mgr Fabre du 24 février 1870 au 4 novembre 1896. - [Avant le 8 septembre 1876: p. 1-11] - p. 1-75.

2. En retournant le cahier dans l'autre sens: Récit de voyage en Europe du 11 juin 1869 au 8 mars 1870 pour le 1er Concile du Vatican. - 136 p.

3. Après ce récit, 5 p. de notes du 2 septembre 1888 au 23 janvier 1891 et du 19 septembre au 3 octobre 1896.

RCD 118 - Véritables motifs de Messieurs et Dames de la Société de NostreDame de Monréal pour la conversion des Sauvages de la Nouvelle France :

Transcription $(90$ p.) de ce texte, ayant appartenue à J. Desautels, curé. Cet écrit, paru en 1643, a reparu en édition photocopique dans: Marie-Claire Daveluy, La Société de Notre-Dame de Montréal, Fides, Montréal et Paris, 1965, après la p. 326.

RCD 119 - Lettre et Journal adressé par Mr Dupuy, Ptre à Monseigneur J. J. Lartigue, Évêque de Montréal, en 1836:

M. Jean-Baptiste Dupuy, ptre, (15 septembre 1804 - 13 octobre 1879) a été, entre autres occupations, missionnaire au Témiscamingue (1836), rédacteur des Mélanges Religieux (1843-1845) et curé de St-Antoine-sur-Richelieu (1858-1877). On consultera sur ce texte ici présenté: Album des familles, ler janvier 1881 et, de l'abbé J.-B. Proulx, A la Baie d'Hudson, Montréal, $1886,285 \mathrm{p}$. Le cahier a $55 \mathrm{p}$.

1. "Lettre et journal adressé par M. Dupuy, ptre, à Monseigneur J.-J. Lartigue Evêque de Montréal, en 1836." -- La Lettre a 8 p. et vient ensuite le "Journal d'un voyage fait à Temiskaming." - 20 juin 1836 au 16 août 1836 - Ce voyage fut fait en compagnie de M. DeBellefeuille, p.s.s. C'était la première expédition missionnaire envoyée dans cette région par $\mathrm{Mgr}$ Lartigue depuis son élévation à l'épiscopat. (Cf. Yvon Charron, p.s.s., Monsieur Charles DeBellefeuille, Missionnaire de l'Outawais (1836-1838) in $R H A F, \mathrm{~V}, \mathrm{n}^{\circ} 2$ (1951): 193-226.)

2. Noms de ceux qui ont fait leur Première communion à Le Passe, les 7 et 8 août 1836; au poste de Temiskaming, l'an 1836, l'an 1837; en 1838, $1^{\circ}$ au poste de Temiscaming, $2^{\circ}$ au poste des Allumettes; à Temiskaming le 23 juin 1839.

3. En 3e p. couverture: "Noms des hommes qui ont conduit les Missionnaires à Temiskaming en 1836; en 1837, jusqu'à Abittibi; en 1838, jusqu'à Temiskaming, Abittibi et au Grand Lac."

RCD 121 - Doit l'Évêché de Montréal à Mgr Vinet (1871):

Livre des comptes payables par l'Evêché à Mgr Vinet et à ses héritiers à partir du 9 janvier 1872 . - Texte aux p. 1, 13, 20, 21, 27, 33, 40, 50, 60 et 70. 


\section{RCD 122 - Voyages de Crespel :}

Voyages/ en Canada/ par le R.P. Emmanuel Crespel, recolet:/ et/ son naufrage sur l'Isle d'anticostie en 1736/ Imprimé à la nouvelle imprimerie,/ 1808. - Sous forme de 8 lettres datées du 10 janvier 1742 au 18 juin 1742 . - 28 p.

\section{RCD 124 et 125 - Montréal - Ordonnances I et II :}

Ordonnances de visites pastorales déposées dans un cahier à onglets. - Vol. I - p. 1-396 et Vol. II - p. 397-651. - Index à la fin du vol. II (1714 (?) - 1871 (?)).

RCD 126 - "Sommaire des mariages, batêmes et sépultures de la paroisse de Montréal depuis son établissement en 1643, et des premières communions depuis 1820 inclus." :

1ère partie: $1643-1873 ; 2 \mathrm{e}$ partie: $1820-1828$. - $20 \mathrm{p}$.

RCD 130 - Transcription de la correspondance de Mgr J.-N. Provencher à Mgr J.-J. Lartigue (1821 - 1839) :

Copie dactylographiée. - Correspondance du 15 juillet 1821 au 6 juillet 1839 . - Original conservé aux Archives de la Chancellerie de l'Archevêché de Montréal, au dossier $\mathbf{n}^{0} 255.109$ : St-Boniface.

RCD 131 - Transcription de la correspondance de Mgr J.-N. Provencher à Mgr Bourget (1831 - 1853) :

Copie dactylographiée - Correspondance du 8 août 1831 au 26 mars 1853.

- Original conservé aux Archives de la Chancellerie de l'Archevêché de Montréal, au dossier $\mathbf{n}^{0}$ 255.109 : St-Boniface.

RCD 132 - Transcription de la correspondance de Mgr J.-N. Provencher à divers personnages $(1836$ - 1849) :

Originaux conservés aux Archives de la Chancellerie de l'Archevêché de Montréal, au dossier $\mathbf{n}^{0}$ 255.109 : St-Boniface.

1. A Mgr l'Evêque de Québec (Joseph Signay) -- 19 janvier 1836. - 5 p.

2. Indult du 29 mai 1836. -1 p.

3. A Mgr Joseph Signay, Ev. de Québec, du 13 octobre 1837, avec une lettre de Mgr Joseph Signay à Mgr J.-J. Lartigue du 21 mars 1837 et une note ajoutée par M. C.-J. Cazeau de la même date. - 6 p.

4. A Monsieur ... - 4 juillet 1837. - 3 p.

5. A M. A.-F. Truteau, ptre. -13 juillet $1841,-4 \mathrm{p}$.

6. Indult du 16 juin 1844 . $-8 \mathrm{p}$.

7. Extrait d'une lettre à Mgr Turgeon, Ev. de Sidyme et Coadjuteur de Québec. -20 avril 1849 . $-1 \mathrm{p}$.

8. A Mgr Prince, Ev. de Martyropolis. - 16 juin 1849. - 7 p.

9. A M. J.-Bte Proulx, ptre. -5 juin $1848 .-4$ p.

10. Lettre de M. J.-Bte Proulx, ptre, à Mgr J.-N. Provencher, Ev. de Juliopolis; - 14 mars 1848.

11. A Mgr de Mazenod, évêque de Marseille. - 29 novembre 1849. - 4 p.

RCD 133 - Liste des prêtres ordonnés pour Montréal (1836 - 1964) :

Dates d'ordinations, noms des prêtres, et références aux sources. -1 p. par année. - par l'auteur de cet Inventaire. 
RCD 134 - Journal de voyage de Mgr J.-J. Lartigue en Europe (1819 - 1820) : Transcription dactylographiée (original) du Journal. - 30 juin $1819 \mathrm{au} 7$ août 1820. - 161 p. - Original conservé aux Archives de la Chancellerie de l'Archevêché de Montréal, au dossier $\mathbf{n}^{0}$ 901.036 : Mgr Lartigue. - Journal de voyage en Europe.

RCD 135 - idem : copie de la transcription.

RCD 137 - Itinéraire du voyage de l'Évêque de Montréal à Rome (1854 - 1856) : Transcription dactylographiée (original) de l'Itinéraire du voyage de $\mathrm{Mgr}$ Bourget à Rome. - 23 octobre 1854 au 29 juillet 1856 . - 17 p. - Original conservé aux Archives de la Chancellerie de l'Archevêché de Montréal au dossier $n^{0}$ 901.056: Mgr Bourget. - Lettres personnelles (1856-1859) et voyages à Rome (1854-1856).

RCD 138 - Liste des prêtres ordonnés pour Montréal (1836 - 1965) :

Texte revu, augmenté et corrigé de RCD 133. - Par l'auteur de cet Inventaire.

RCD 139 - Actes du 1er Synode de Montréal (1863) :

Texte de la convocation du 22 juin 1863 et procès-verbaux des séances. - $66 \mathrm{p}$.

RCD 140 - Mariages - Promesses de la partie protestante (1844 - 1887) : Cahier à onglets de 120 documents avec Index alphabétique. [Avant 1877: 74 documents]. 